\title{
Expression of programmed death ligand 1 (PD-L1) is associated with poor prognosis in human breast cancer
}

\author{
S. Muenst • A. R. Schaerli • F. Gao $\cdot$ S. Däster $\cdot$ E. Trella $\cdot$ R. A. Droeser $\cdot$ \\ M. G. Muraro · P. Zajac $\cdot$ R. Zanetti $\cdot$ W. E. Gillanders $\cdot$ W. P. Weber \\ S. D. Soysal
}

Received: 24 April 2014/Accepted: 29 April 2014/Published online: 20 May 2014

(c) Springer Science+Business Media New York 2014

\begin{abstract}
Recent studies in multiple epithelial cancers have shown that the inhibitory receptor programmed cell death 1 (PD-1) is expressed on tumor-infiltrating lymphocytes and/or programmed death ligand 1 (PD-L1) is expressed on tumor cells, suggesting that antitumor immunity may be modulated by the PD-1/PD-L1 signaling pathway. In addition, phase 1 clinical trials with monoclonal antibodies targeting PD-1 or PD-L1 have shown promising results in several human cancers. The purpose of this study was to investigate the impact of PD-L1 expression in human breast cancer specimens. We conducted an immunohistochemistry study using a tissue microarray encompassing 650 evaluable formalin-fixed breast cancer cases with detailed clinical annotation and outcomes data. PD-L1 was expressed in 152 (23.4\%) of the 650 breast cancer specimens. Expression was significantly associated with age, tumor size, AJCC primary tumor classification, tumor grade, lymph node status, absence of ER expression, and high Ki-67 expression. In univariate analysis, PD-L1 expression was associated with a significantly worse OS. In
\end{abstract}

S. Muenst and A. R. Schaerli have contributed equally to this study.

S. Muenst ( $\square)$

Institute of Pathology, University Hospital Basel,

Schönbeinstrasse 40, CH-4032 Basel, Switzerland

e-mail: muensts@uhbs.ch

A. R. Schaerli · S. Däster · R. A. Droeser ·

W. P. Weber - S. D. Soysal

Department of Surgery, University Hospital Basel, Basel,

Switzerland

e-mail: a.schaerli@hotmail.ch

F. Gao

Division of Biostatistics, Washington University School of

Medicine, St. Louis, MO, USA multivariate analysis, PD-L1 expression remained an independent negative prognostic factor for OS. In subset analyses, expression of PD-L1 was associated with significantly worse OS in the luminal B HER2 ${ }^{-}$subtype, the luminal B HER2 ${ }^{+}$subtype, the HER2 subtype, and the basal-like subtype. This is the first study to demonstrate that PD-L1 expression is an independent negative prognostic factor in human breast cancer. This finding has important implications for the application of antibody therapies targeting the PD-1/PD-L1 signaling pathway in this disease.

Keywords PD-L1 . PD-1 - Tumor immunology breast cancer Prognostic factor TIL

\section{Introduction}

$\mathrm{T}$ cells play a critical role in the human immune system, recognizing foreign antigens and orchestrating an effective immune reaction. However, despite the ability to recognize tumor-associated antigens, $\mathrm{T}$ cells are often unable to

\footnotetext{
S. Däster · E. Trella · R. A. Droeser - M. G. Muraro

P. Zajac · S. D. Soysal

Institute of Surgical Research and Hospital Management (ICFS) and Department of Biomedicine, University of Basel, Basel, Switzerland

R. Zanetti

Department of Gynecology, University Hospital Basel, Basel, Switzerland

W. E. Gillanders

Department of Surgery, Washington University School of Medicine, St. Louis, MO, USA
} 
mediate tumor regression [1]. The interaction between $\mathrm{T}$ cells and antigen-presenting cells (APC) is complex and involves the $\mathrm{T}$ cell receptor and multiple co-stimulatory receptors, which exert both activating and inhibitory stimuli to the $\mathrm{T}$ cell. The interplay between activating and inhibitory signals is especially important in modulating the immune system's ability to distinguish self from nonself and to prevent autoimmune reactions [2, 3]. Programmed cell death 1 (PD-1), a member of the CD28/CTLA-4 family of co-stimulatory receptors, conveys an inhibitory signal to the $\mathrm{T}$ cell and thus impedes immune responses. PD-1 contributes to the immune tolerance of self antigens by peripheral T cells $[2,3]$. PD-1 is expressed on natural killer cells, dendritic cells (DCs), activated monocytes as well as on B cells, and T cells [4-6]. Persistent high-level PD-1 expression on antigen-experienced $\mathrm{CD} 8^{+} \mathrm{T}$ cells leads to a $\mathrm{CD}^{+} \mathrm{T}$ cell phenotype characterized by impaired effector function and persistent expression of inhibitory receptors, termed "T cell exhaustion" [7]. PD-1 has two ligands, programmed death ligand 1 (PD-L1) and programmed death ligand 2 (PD-L2) [8]. PD-L1 is expressed not only on APC, DCs, as well as on activated monocytes and B cells, but also on nonlymphoid tissues of different organs [8, 9]. Of note, expression of PD-L1 has been discovered in a variety of epithelial cancers such as non-small cell lung carcinoma (NSCLC) [10], pancreatic cancer [11], esophageal cancer [12], squamous cell carcinomas of the head and neck, and renal cell carcinoma (RCC) [13]. Recent evidence suggests that activation of the PD-1/PD-L1 pathway represents one mechanism allowing tumors to elude the host's immune system $[14,15]$. The expression of PD-L1 has been associated with poor prognosis and fewer tumorinfiltrating lymphocytes (TIL) in pancreatic cancer and RCC [11, 16, 17]. The exertion of this pathway by cancer cells might also explain why, in ongoing studies investigating cancer vaccinations, there is no tumor growth control despite the induction of cancer-specific T cells [18-20]. These observations provide strong rationale for targeting PD-1 or its ligand PD-L1 with antibodies capable of inhibiting this pathway. Preclinical murine and ex vivo studies with anti-PD-1 antibodies have shown promising results, demonstrating that $\mathrm{PD}-1$ blockade in combination with antitumor immunotherapy can prolong survival of mice inoculated with live melanoma cells [21]. In addition, PD-1 blockade results in an increase of antigen-specific cytotoxic $\mathrm{T}$ cells in melanoma in vitro [21-23]. Based on these data, early phase human clinical trials targeting the PD-1/PD-L1 pathway are currently under way for multiple human cancers [25, 28]. Phase I clinical trials have been conducted using the human anti-PD-1 antibody (BMS936558 also known as Nivolumab) demonstrating objective responses (response rates 6-38\%) in patients with
NSCLC, advanced melanoma, RCC, and castration resistant prostate cancer [24-27].

A multicenter phase I trial with a human anti-PD-L1 antibody (BMS-936559) in advanced human cancers showed durable tumor regression (objective response rate 6-17\%) and prolonged stabilization of disease [28]. In this clinical trial, success was documented in cancers that have previously been considered resistant to immunotherapy, such as NSCLC and RCC. Some of these responses were durable, suggesting that targeting the PD-1/PD-L1 signaling pathway is likely to develop into an important treatment modality for patients with advanced cancer.

Since breast cancer is the most common carcinoma in women, and the second most common cause of cancer death among women worldwide [29], defining the importance of the PD-1/PD-L1 signaling pathway in breast cancer is of significant clinical relevance.

Although breast cancer is commonly thought to be less immunogenic than melanoma or RCC, there is increasing evidence of a dynamic crosstalk between the immune system and breast cancer. Recent studies have revealed the presence of regulatory $\mathrm{T}$ cells in peripheral blood as well as in the tumors of breast cancer patients [30, 31]. Furthermore, inhibitory molecules of the CD28 receptor family are upregulated on breast cancer-specific $\mathrm{T}$ cells [32-34], and PD-L1 is expressed on breast cancer cells [34].

We have recently shown that the presence of PD$1+$ tumor infiltrating lymphocytes (TIL) is associated with adverse clinicopathological features and is an independent negative prognostic factor in human breast cancer [35]. We also found a significant difference in the presence of PD$1+$ TIL in the intrinsic breast cancer subtypes, as specified by the St. Gallen consensus conference [35, 36].

Ghebeh et al. [34] demonstrated that PD-L1 is expressed in primary breast cancer specimens (either on the cancer cells themselves or by TIL) yet not in healthy adjoining tissue from the same breast. They found PD-L1 expression by tumor cells in $34 \%$ of their cases and showed that PDL1 is significantly correlated with grade 3 tumors [33, 34, 37]. In a second study, the same authors also demonstrated a correlation between expression of PD-L1by tumor cells and a high expression of $\mathrm{Ki}-67$ as well as absence of estrogen-receptor (ER) and progesterone-receptor (PR) expression [37].

These data strongly suggest a potential association between activation of the PD-1/PD-L1 pathway in breast cancer and poor prognosis. The promising results of the phase I clinical trials targeting this pathway suggest that these therapies could be successful in human breast cancer, particularly in patients with clinically advanced disease, or in patients with ER-and/or PR-negative subtypes, which generally have a poorer prognosis $[36,38]$. 
Table 1 Basic demographic data for 650 evaluable breast cancer cases

\begin{tabular}{|c|c|c|}
\hline \multicolumn{2}{|l|}{$\begin{array}{l}\text { Mean tumor size }(\mathrm{mm}) \\
\quad \pm \text { standard deviation }(\mathrm{SD})\end{array}$} & $29.6 \pm 16.8$ \\
\hline \multirow{2}{*}{$\begin{array}{l}\text { Mean age at diagnosis (years) } \\
\pm \text { standard deviation (SD) }\end{array}$} & \multicolumn{2}{|c|}{$63.8 \pm 14.2$} \\
\hline & $\begin{array}{l}\text { Number } \\
(n)\end{array}$ & $\begin{array}{l}\text { Percent } \\
(\%)\end{array}$ \\
\hline \multicolumn{3}{|l|}{ Tumor stage } \\
\hline pT1 & 181 & 27.9 \\
\hline pT2 & 350 & 53.8 \\
\hline pT3 & 35 & 5.4 \\
\hline pT4 & 84 & 12.9 \\
\hline \multicolumn{3}{|l|}{ Lymph node involvement } \\
\hline pNO & 355 & 54.7 \\
\hline pN1 & 226 & 34.8 \\
\hline $\mathrm{pN} 2$ & 68 & 10.5 \\
\hline \multicolumn{3}{|l|}{ Tumor grade } \\
\hline 1 & 143 & 22 \\
\hline 2 & 259 & 39.8 \\
\hline 3 & 248 & 38.2 \\
\hline \multicolumn{3}{|l|}{ Histologic subtype } \\
\hline Invasive ductal & 482 & 76.4 \\
\hline Invasive lobular & 72 & 11.4 \\
\hline Mucinous & 23 & 3.6 \\
\hline Apocrine & 3 & 0.5 \\
\hline Cribriform & 14 & 2.2 \\
\hline Papillary & 8 & 1.3 \\
\hline Medullary & 29 & 4.6 \\
\hline \multicolumn{3}{|l|}{ Intrinsic subtype } \\
\hline $\begin{array}{l}\text { Luminal A }\left(\mathrm{ER}^{+} \text {and/or }\right. \\
\left.\mathrm{PR}^{+}, \mathrm{HER}^{-}, \mathrm{Ki}-67<14 \%\right)\end{array}$ & 83 & 12.8 \\
\hline $\begin{array}{l}\text { Luminal B (HER2-negative) }\left(\mathrm{ER}^{+}\right. \\
\left.\text {and/or } \mathrm{PR}^{+}, \mathrm{HER}^{-}, \mathrm{Ki}-67 \geq 14 \%\right)\end{array}$ & 309 & 47.7 \\
\hline $\begin{array}{l}\text { Luminal B (HER2-positive) }\left(\mathrm{ER}^{+}\right. \\
\left.\text {and/or } \mathrm{PR}^{+}, \mathrm{HER}^{+}\right)\end{array}$ & 73 & 11.3 \\
\hline HER2 type $\left(\mathrm{ER}^{-}\right.$or $\left.\mathrm{PR}^{-}, \mathrm{HER}^{+}\right)$ & 56 & 8.6 \\
\hline Basal-like $\left(\mathrm{ER}^{-}, \mathrm{PR}^{-}, \mathrm{HER} 2^{-}\right)$ & 127 & 19.6 \\
\hline
\end{tabular}

However, recent data from a study looking at in situ tumor PD-L1 mRNA expression suggests that PD-L1 expression may be associated with an increase in the number of TIL, suggesting an association with improved prognosis [39]. Clearly, further study is required to define the role of PD-L1 expression in breast cancer.

To further explore the impact of PD-L1 expression in human breast cancer, we conducted an immunohistochemistry study using a breast cancer tissue microarray (TMA) encompassing a total of 1,460 formalin-fixed breast cancer cases with detailed clinical annotation and outcomes data. The aim of our study was to investigate the expression of PD-L1 by breast cancer cells and its association with the expression of PD-1 by TIL as well as with clinicopathological parameters, with a particular focus on any potential association with prognosis. The data is reported according to the reporting recommendations for tumor marker prognostic studies (remark) [40].

\section{Materials and methods}

Tissue microarray

We used a TMA encompassing 1,460 breast cancer tissue punches from formalin-fixed and paraffin-embedded tumor samples collected from patients diagnosed with primary breast cancer between 1985 and 2007 at the Institute for Pathology, University of Basel and the Viollier Institute in Basel, Switzerland. Of these 1,460 tissue punches, due to the tissue loss or lack of breast cancer tissue on individual punches, a total of 650 were evaluable for our study. The tissue samples were brought into a TMA format as previously described [41]. Histopathologic data were obtained from the pathology reports, and raw patient survival data was obtained from the Cancer Registry of Basel or from the patient's attending physician. Retrieval of tissue and clinical data was performed according to the regulations of the local institutional review boards and data safety laws with specific regard to ethical standards and patient confidentiality. The mean follow-up time was 65 months (range 1-174 months), and the mean age of the patients at diagnosis was 64 years (range 27-101 years). Demographic information of the patients is given in Table 1.

\section{Immunohistochemistry}

For immunohistochemical staining, 4- $\mu \mathrm{m}$ sections of the TMA blocks were incubated for $30 \mathrm{~min}$ with a prediluted rabbit-anti-human PD-L1 polyclonal antibody (Abcam, Cambridge, UK) after heat-induced antigen retrieval with Cell Conditioning Solution (Ventana Medical Systems, Tuscon, AZ, USA). Standard DAB-technique (Optiview DAB IHC Detection Kit, Ventana Medical Systems, Tuscon, AZ, USA) was employed for immunostaining. Counterstaining was performed with hematoxylin solution. Since PD-L1 is expressed on the cell membrane as well as the endomembrane system, membranous as well as cytoplasmic staining was considered positive (Fig. 1). Frequency and staining intensity of PD-L1 by tumor cells were analyzed, and PD-L1 expression was quantified using the modified Histo-score (H-score) [42], with a range of possible scores from 0 to 300. PD-L1 expression was dichotomized into two groups according to the frequency 
Table 2 Association between PD-L1 expression and clinicopathological parameters

\begin{tabular}{llll}
\hline Clinicopathologic parameter & $\begin{array}{l}\text { PD-L1- } \\
\text { positive }\end{array}$ & $\begin{array}{l}\text { PD-L1- } \\
\text { negative }\end{array}$ & $\begin{array}{l}p \text { - } \\
\text { value }\end{array}$ \\
\hline $\begin{array}{l}\text { Mean tumor size }(\mathrm{mm}) \pm \mathrm{SD} \\
\text { Mean age at diagnosis }\end{array}$ & 35.1 & 62.9 & $<.0001$ \\
$\quad 28.1$ & 62.9 & $<.0001$ \\
\hline years $) \pm \mathrm{SD}$ & & & \\
\hline
\end{tabular}

\begin{tabular}{|c|c|c|c|c|c|}
\hline & $(n)$ & $(\%)$ & $(n)$ & $(\%)$ & $<.0001$ \\
\hline \multicolumn{6}{|c|}{ Tumor stage } \\
\hline pT1 & 25 & 13.8 & 156 & 86.2 & \\
\hline pT2 & 82 & 23.4 & 268 & 76.6 & \\
\hline pT3 & 15 & 42.9 & 20 & 57.1 & \\
\hline pT4 & 30 & 35.7 & 54 & 64.3 & \\
\hline \multicolumn{5}{|c|}{ Lymph node involvement } & $<.0001$ \\
\hline $\mathrm{pNO}$ & 65 & 18.3 & 290 & 81.7 & \\
\hline $\mathrm{pN} 1$ & 52 & 23.0 & 174 & 77.0 & \\
\hline $\mathrm{pN} 2$ & 34 & 50.0 & 34 & 50.0 & \\
\hline \multicolumn{5}{|c|}{ Tumor grade } & 0.0007 \\
\hline 1 & 27 & 18.9 & 116 & 81.1 & \\
\hline 2 & 47 & 18.1 & 212 & 81.9 & \\
\hline 3 & 78 & 31.4 & 170 & 68.6 & \\
\hline \multicolumn{5}{|c|}{ Estrogen-receptor } & 0.0020 \\
\hline $\mathrm{ER}^{+}$ & 92 & 20.1 & 365 & 79.9 & \\
\hline $\mathrm{ER}^{-}$ & 60 & 31.4 & 131 & 68.6 & \\
\hline \multicolumn{5}{|l|}{ HER 2} & 0.0237 \\
\hline $\mathrm{HER}^{+}{ }^{+}$ & 40 & 31.0 & 89 & 69.0 & \\
\hline HER $2^{-}$ & 112 & 21.6 & 407 & 78.4 & \\
\hline \multicolumn{5}{|l|}{ Ki67 } & 0.0043 \\
\hline $\mathrm{Ki} 67^{+}$ & 136 & 25.9 & 389 & 74.1 & \\
\hline $\mathrm{Ki}^{-} 7^{-}$ & 16 & 13.6 & 102 & 86.4 & \\
\hline \multicolumn{5}{|l|}{ PD-1 } & $<.0001$ \\
\hline PD-1 ${ }^{+}$ & 69 & 66.3 & 35 & 33.7 & \\
\hline PD-1 ${ }^{-}$ & 83 & 15.2 & 463 & 84.8 & \\
\hline
\end{tabular}

distributions of the H-scores, using a cut-off score of $\geq 100$ (H-score $\quad 0-99=$ negative/low expression, and 100-300 = positive expression). The staining intensity of ER, PR, and HER2 was scored as described previously [43].

Flow cytometry of human breast cancer specimens

Tumor cells suspension was derived from freshly excised human breast cancer specimens, obtained from consenting patients undergoing surgical treatment at Basel University Hospital. Tissues were cut into small pieces $(1 \mathrm{~mm} \times$ $1 \mathrm{~mm}$ ) and digested in serum-free DMEM (GIBCO), supplemented with collagenase IV (Worthington, New Jersey, USA) and DNAse I (Sigma-Aldrich, St.Louis, Missouri, USA) at $37{ }^{\circ} \mathrm{C}$ for $60 \mathrm{~min}$. After digestion, single cell
Table 3 Association between PD-L1 expression and breast cancer intrinsic subtype

\begin{tabular}{|c|c|c|c|c|c|}
\hline \multirow[t]{2}{*}{ Intrinsic subtype } & \multicolumn{2}{|c|}{$\begin{array}{l}\text { PD-L1- } \\
\text { positive }\end{array}$} & \multicolumn{2}{|c|}{$\begin{array}{l}\text { PD-L1- } \\
\text { negative }\end{array}$} & \multirow{2}{*}{$\begin{array}{l}p \text { - } \\
\text { value } \\
0.5340\end{array}$} \\
\hline & $(n)$ & $(\%)$ & $(n)$ & $(\%)$ & \\
\hline $\begin{array}{l}\text { Luminal A } \\
\left(\mathrm{ER}^{+} \text {and/or } \mathrm{PR}^{+}, \mathrm{HER}^{-}, \mathrm{Ki}^{-}\right. \\
\quad 67<14 \%)\end{array}$ & 10 & 12.1 & 73 & 87.9 & \\
\hline $\begin{array}{l}\text { Luminal B (HER2-negative) } \\
\left(\mathrm{ER}^{+} \text {and/or } \mathrm{PR}^{+}, \mathrm{HER}^{-}, \mathrm{Ki}^{-}\right. \\
\quad 67 \geq 14 \%)\end{array}$ & 63 & 20.4 & 246 & 79.6 & \\
\hline $\begin{array}{l}\text { Luminal B (HER2-positive) } \\
\left(\mathrm{ER}^{+} \text {and/or } \mathrm{PR}^{+}, \mathrm{HER}^{+}\right)\end{array}$ & 21 & 28.8 & 52 & 71.2 & \\
\hline HER2 type $\left(\mathrm{ER}^{-}, \mathrm{PR}^{-}, \mathrm{HER} 2^{+}\right)$ & 19 & 33.9 & 37 & 66.1 & \\
\hline Basal-like $\left(\mathrm{ER}^{-}, \mathrm{PR}^{-}, \mathrm{HER} 2^{-}\right)$ & 39 & 30.7 & 88 & 69.3 & \\
\hline
\end{tabular}

Table 4 Univariate analyses for all cases, and by intrinsic subtype, for the effect of PD-L1 expression on overall survival

\begin{tabular}{lcc}
\hline PD-L1 expression, all cases & Hazard ratio (95 \% CI) & $p$ value \\
\hline PD-L1-positive & $4.430(3.424-5.731)$ & $<.0001$ \\
PD-L1 expression, by intrinsic subtype & \\
Luminal A & $2.693(0.743-9.764)$ & 0.1317 \\
Luminal B (HER2 $\left.{ }^{-}\right)$ & $3.888(2.651-5.704)$ & $<.0001$ \\
Luminal B (HER2 $\left.{ }^{+}\right)$ & $5.172(2.412-11.094)$ & $<.0001$ \\
HER2 type & $2.834(1.244-6.452)$ & 0.0131 \\
Basal-like & $4.973(2.935-8.426)$ & $<.0001$ \\
\hline
\end{tabular}

suspension was obtained by filtration through $100-\mu \mathrm{m}$ - and $70-\mu \mathrm{m}$-mesh filter. Surface phenotype was determined by flow cytometry using the following antibodies: phycoerythrin-(PE) labeled anti-PD-L1 (clone MIH1), fluorescein isothiocyanate- (FITC) labeled anti-CD3 (clone SK7), allophycocyanin-(APC) labeled anti-CD4 (clone SK3), APClabeled anti-CD8 (clone RPA-T8), FITC-labeled anti-CD90 (clone 5E10), and APC-labeled anti-EpCAM (clone EBA1). All the antibodies were purchased from BD Bioscience (San Jose, USA). Propidium iodide (PI, $0.5 \mu \mathrm{g} / \mathrm{ml}$ ) was added to the samples prior to analysis. Relative fluorescence intensities were measured using a BD FACScalibur flow cytometer (BD Biosciences) following exclusion of dead cells based on PI incorporation. Analysis was performed using FlowJo software (Tree Star, Portland, OR).

Statistical analysis

The distributions of patient and clinical characteristics between tumors expressing PD-L1 and those negative for PD-L1 were compared using Chi-square test, Wilcoxon rank-sum test, or two-sample $t$ test, as appropriate. Overall survival (OS) was defined as the time from the first 

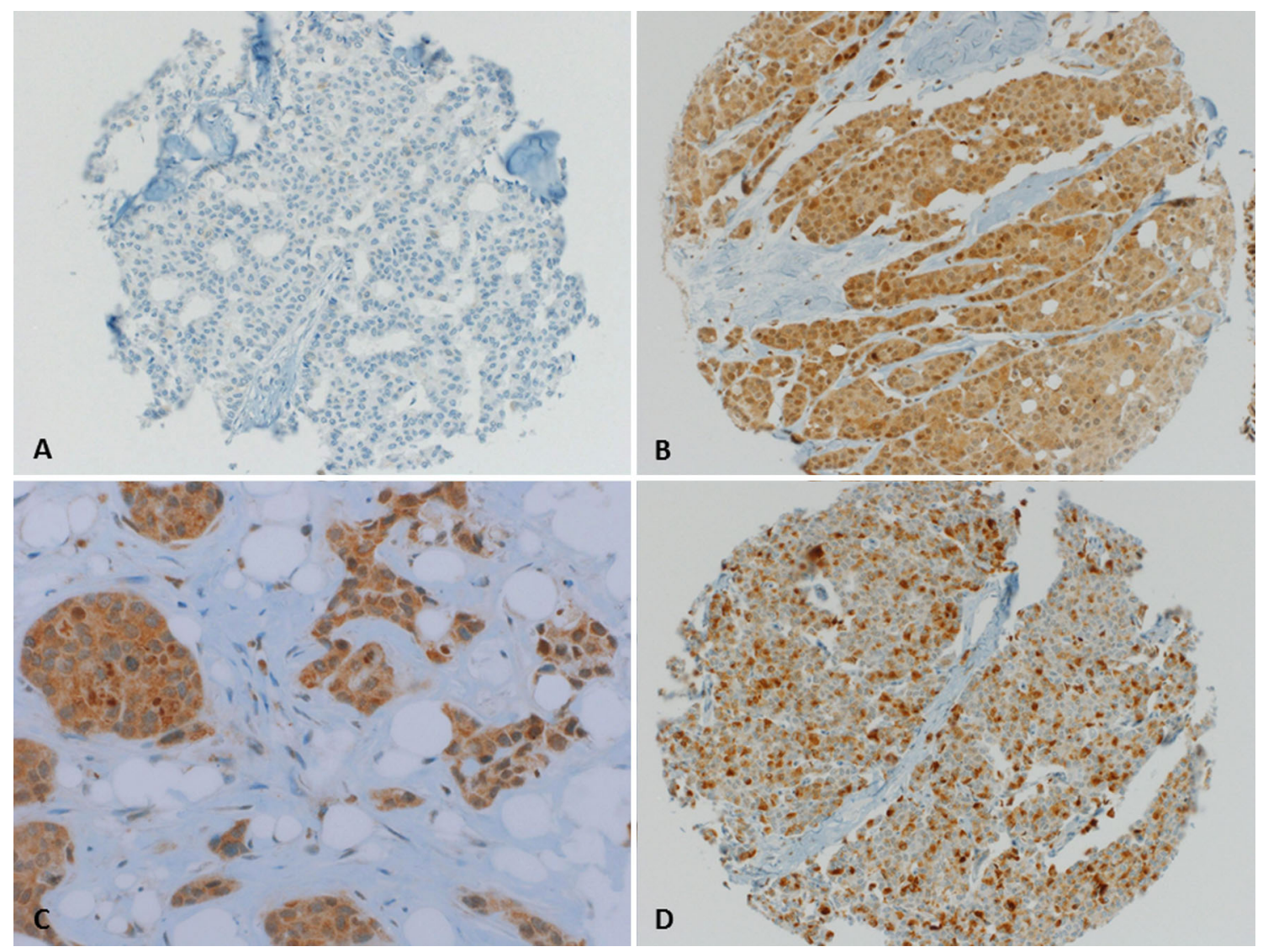

Fig. 1 Representative photographs of PD-L1 expression in breast cancer tissue punches a Tissue punch negative for PD-L1 expression. Magnification $\times 200$ b Tissue punch with strong PD-L1 expression in

operation to death due to any cause. Survivors were censored at the date of last contact. Survival curves by expression of PD-L1 were estimated using the KaplanMeier product-limit method and compared by log-rank test. Univariate Cox proportional hazard models were fit to identify factors significantly related to OS. To assess whether the expression of PD-L1 by tumor cells was an independent predictor of survival, a multivariate Cox model was constructed to adjust other patient/clinical characteristics that were significant in the univariate analyses. Two-way interaction terms between expression of PD-L1 and other factors in the multivariate Cox model were also assessed. All analyses were two-sided and significance was set at a $p$-value of 0.05 . Statistical analyses were performed using SAS (SAS Institutes, Cary, NC).

\section{Results}

Expression of PD-L1 by tumor cells was present in a total of $152(23.4 \%)$ of the 650 evaluable primary breast cancers. The expression of PD-L1 was significantly associated with age, tumor size, AJCC primary tumor classification, tumor
$100 \%$ of cells. Magnification $\times 200$ c Same tissue punch as in b magnification $\times 400$. d Tissue punch with strong PD-L1 expression in $40 \%$ of cells. Magnification $\times 200$

grade, and lymph node status (Table 2). The expression of PD-L1 was positively associated with Ki-67 expression ( $p=0.0043)$, and HER 2 expression $(p=0.0237$, Table 2$)$, and negatively associated with ER expression $(p=0.0020)$ (Table 2). There was no significant association with PR expression $(p=0.1893)$. There was also no significant difference of PD-L1 expression among the different intrinsic subtypes of breast cancer, as defined by the St Gallen consensus conference (Table 3) [36]. The breast cancer intrinsic subtypes were originally defined by gene expression profiling $[44,45]$ but can be approximated using immunohistochemistry for ER, PR, Ki-67, and HER2 [36, 46]. These subtypes are known to have differing epidemiological risk factors, prognosis, and response to therapy [36]. Interestingly, there was a strong correlation between the expression of PD-L1 by tumor cells and the presence of PD-1-positive TIL $(p<0.001)$.

In univariate survival analyses, breast cancer cases expressing PD-L1 had a significantly worse OS (HR = $4.430, p<0.0001$, Table 4 and Fig. 2). In subset analyses by intrinsic subtype, the expression of PD-L1 was associated with decreased OS in the luminal B HER2 ${ }^{-}$subtype, $(\mathrm{HR}=3.888, p<0.0001)$, the luminal B HER $2^{+}$subtype 
Fig. 2 a Kaplan-Meier survival curve for overall survival depending on the expression of PD-L1 (univariate analysis) b-f Kaplan-Meier survival curves for overall survival depending on the expression of PD-L1 for the indicated breast cancer intrinsic subtypes
(A) All

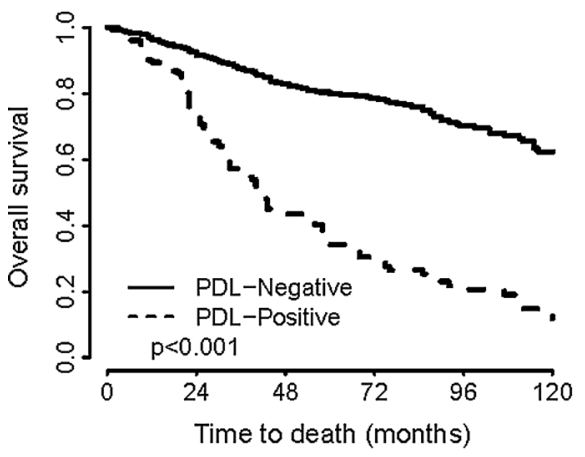

(C) Lum B, Her2-

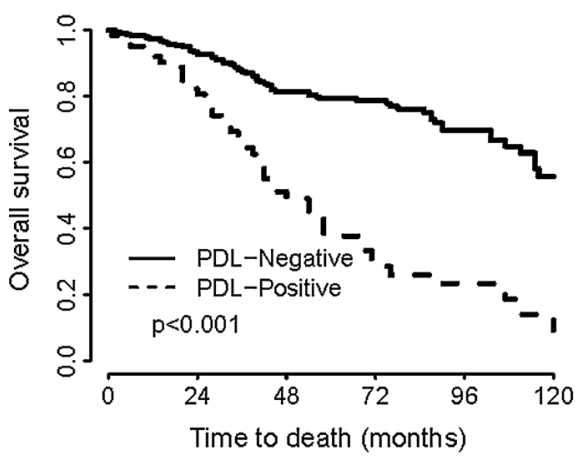

(E) Her2+

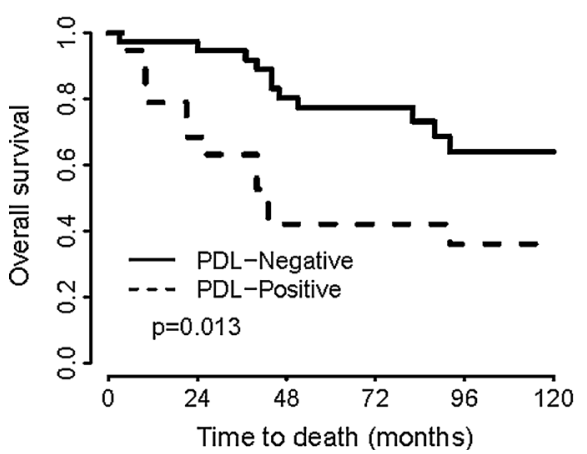

(B) $\operatorname{Lum} A$

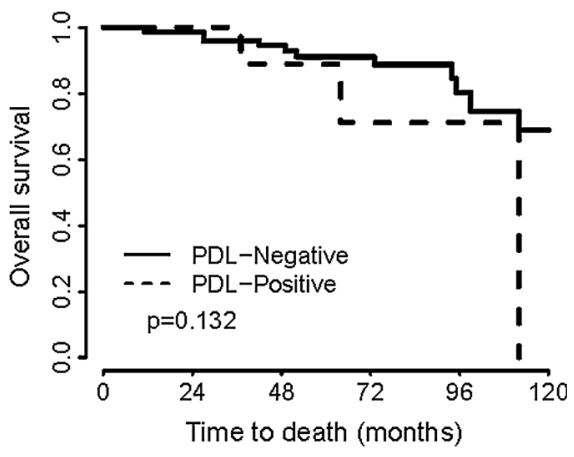

(D) Lum B, Her2+

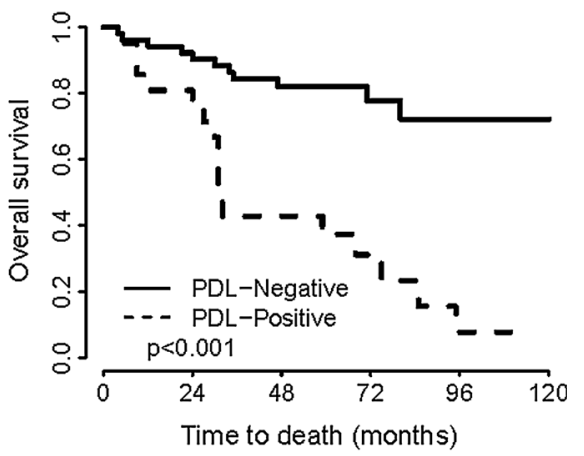

(F) Basal-like

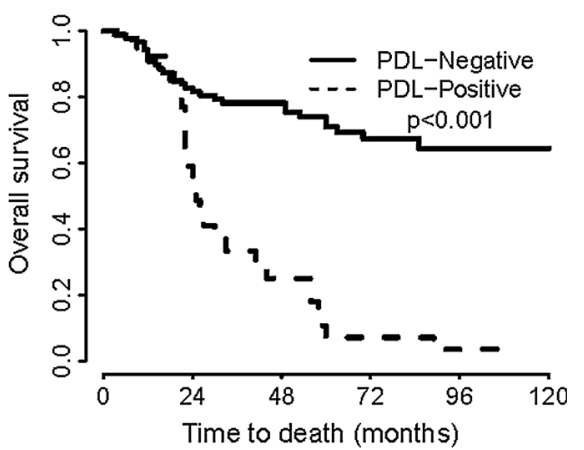

$(\mathrm{HR}=5.127, p<0.0001)$, the HER2 subtype $(\mathrm{HR}=2.834$, $p=0.0131)$, and the basal-like subtype $(\mathrm{HR}=4.973$, $p<0.0001$ ) (Table 4 and Fig. 2). Of note, there was no association with OS in the luminal A subtype. In multivariate analysis, after adjusting for age, grade, tumor size, lymph node status, and intrinsic subtype, the expression of PD-L1 proved to be an independent negative prognostic factor for OS $(\mathrm{HR}=3.063, p<0.0001)$ (Table 5).

In a small subset of 14 cases (9.2\%), we also detected PD-L1 expression on TIL.

To investigate the expression of PD-L1 in the tumor microenvironment in more detail, we performed flow cytometry of cells freshly isolated from four human breast cancers (Fig. 3). While two of the samples were negative for PD-L1, two (one stage I and one stage IV cancer) showed a mean of $2.55 \%$ of PD-L1-positive cells (2.6-2.5\%, respectively). In the stage IV cancer, $2 \%$ of the cells were $\mathrm{EpCAM}^{+} / \mathrm{PD}-\mathrm{L} 1^{+}$, while PD-L1 expression could not be detected on $\mathrm{CD} 4^{+}$or $\mathrm{CD} 8^{+} \mathrm{T}$ cells. In the stage I cancer biopsy, no EpCAM ${ }^{+} / \mathrm{PD}-\mathrm{L1}^{+}$cells could be detected, but instead, $\mathrm{CD} 4^{+} / \mathrm{PD}-\mathrm{L} 1^{+}$and $\mathrm{CD} 8^{+} / \mathrm{PD}-\mathrm{L} 1^{+}$ cells (1-1.8\% of all cells, respectively) (Fig. 3).

\section{Discussion}

We found PD-L1 expression in $23.4 \%$ of primary breast cancer specimens. In a study analyzing tissue from 44 
Table 5 Multivariate analysis for the effect of clinicopathologic parameters and PD-L1 expression on overall survival

\begin{tabular}{|c|c|c|}
\hline Clinicopathologic parameter & Hazard ratio $(95 \% \mathrm{CI})$ & $p$-value \\
\hline Age (per 1 year) & $1.033(1.022-1.044)$ & $<.0001$ \\
\hline \multicolumn{3}{|l|}{ Tumor stage } \\
\hline pT1 (reference) & 1 & \\
\hline pT2 & $1.622(1.084-2.427)$ & 0.0186 \\
\hline pT3 & $1.621(0.869-3.026)$ & 0.1290 \\
\hline pT4 & $2.185(1.334-3.578)$ & 0.0019 \\
\hline \multicolumn{3}{|l|}{ Lymph node involvement } \\
\hline pN1 (reference) & 1 & \\
\hline pN1 & $1.452(1.071-1.968)$ & 0.0165 \\
\hline pN2 & $1.921(1.283-2.875)$ & 0.0015 \\
\hline \multicolumn{3}{|l|}{ Tumor grade } \\
\hline BRE grade 1 (reference) & 1 & \\
\hline 2 & $1.874(1.201-2.923)$ & 0.0057 \\
\hline 3 & $2.537(1.604-4.014)$ & $<.0001$ \\
\hline \multicolumn{3}{|l|}{ PD-L1 expression, all cases } \\
\hline PD-L1-negative (reference) & 1 & \\
\hline PD-L1-positive & $3.063(2.318-4.047)$ & $<.0001$ \\
\hline \multicolumn{3}{|c|}{ PD1 expression, by intrinsic subtype } \\
\hline Luminal A (reference) & 1 & \\
\hline Luminal B (HER2 ${ }^{-}$) & $1.542(0.874-2.724)$ & 0.1352 \\
\hline Luminal B $\left(\right.$ HER2 $\left.^{+}\right)$ & $1.484(0.764-2.882)$ & 0.2437 \\
\hline Her2 type & $1.091(0.530-2.247)$ & 0.8123 \\
\hline Basal-like & $2.394(1.294-4.430)$ & 0.0054 \\
\hline
\end{tabular}

patients, Ghebeh et al. [34] reported PD-L1 expression in $34 \%$ of breast cancers. These results are comparable to our findings, although Ghebeh et al. investigated only patients with invasive ductal carcinoma, and used a different antibody clone and a different scoring system, assessing any expression of PD-L1 by tumor cells as positive. These differences in experimental technique may account for the slightly higher percentage of positive specimens.

Schalper et al. [39] reported PD-L1 mRNA expression in $58 \%$ of their breast cancer specimens. The increase frequency of expression might be explained by the fact that PD-L1 mRNA expression may not always correlate with actual protein expression.

Consistent with our results, Ghebeh et al. [34] also reported an association between PD-L1 expression and higher tumor grade, HER2 expression, and absence of ER expression, however, no significant correlation with lymph node status and patient age was found. A subsequent study by the same group with a total of 68 cases, confirmed these associations and also demonstrated an association between PD-L1expression in breast cancer specimens and high Ki67 expression [37], which we could confirm in our larger patient cohort. The finding that PD-L1 expression in breast cancer specimens is associated with larger and more aggressive tumors as well as with positive lymph node status could indicate that activation of the PD-1/PD-L1 pathway may help these tumors evade antitumor immune responses and consequently proliferate and spread more rapidly.

Soliman et al. [47] investigated their PD-L1 expression in six breast cancer cell lines by flow cytometry. They found the highest expression in cell lines of the basal-like intrinsic subtype, which supports our finding of an association between PD-L1 expression and poor prognosis in this subtype. Soliman et al. [47] also analyzed 61 breast cancer specimens for PD-L1 protein expression. Although there was a suggestion that PD-L1 expression is associated with positive lymph node status, they could not establish a statistically significant association with any clinicopathological parameters.

The present study is the first to show that PD-L1 protein expression in breast cancer specimens is associated with poor prognosis in breast cancer.

In contrast, the study by Schalper et al. [39] looking at in situ PD-L1 mRNA expression on two sets of breast cancer TMAs, found that PD-L1 mRNA expression was significantly associated with disease-free survival, and also suggested an association (that did not reach statistical significance) with longer disease-specific survival in a cohort of 358 patients. They also found that PD-L1 mRNA expression by breast cancer cells was associated with increased TIL [39]. Similar results were reported by the same group in a study analyzing NSCLC, where PD-L1 mRNA or protein expression by tumor cells was significantly associated with better outcome [48]. In their discussion, the authors suggest that expression of PD-L1 by tumor cells might represent antigen-induced antitumor immune pressure, resulting in recruitment of TIL to the tumor site, where they induce a partial antitumor effect. Recent studies support this notion by reporting an association between PD-L1 protein expression and longer survival in metastatic melanoma, [49] merkel cell carcinoma [50], and colorectal carcinoma [51].

We hypothesize that the expression of PD-L1 by tumor cells can contribute to impaired function of TIL, impeding antitumor immunity. This would explain why PD-L1 expression is associated with poor prognosis in our study of human breast cancers, consistent with multiple studies looking at different human cancers such as melanoma, renal, urothelial, gastric, lung, and colorectal cancer [38, 52-55]. This hypothesis is also consistent with the results of recent phase I clinical trials where targeting the PD-1/ PD-L1 pathway resulted in durable tumor regression and stabilization of disease in multiple human epithelial cancers $[25,27,28]$. The inconsistent results related to the association of PD-L1 expression with clinical outcome in some of these cancers could be based on different 

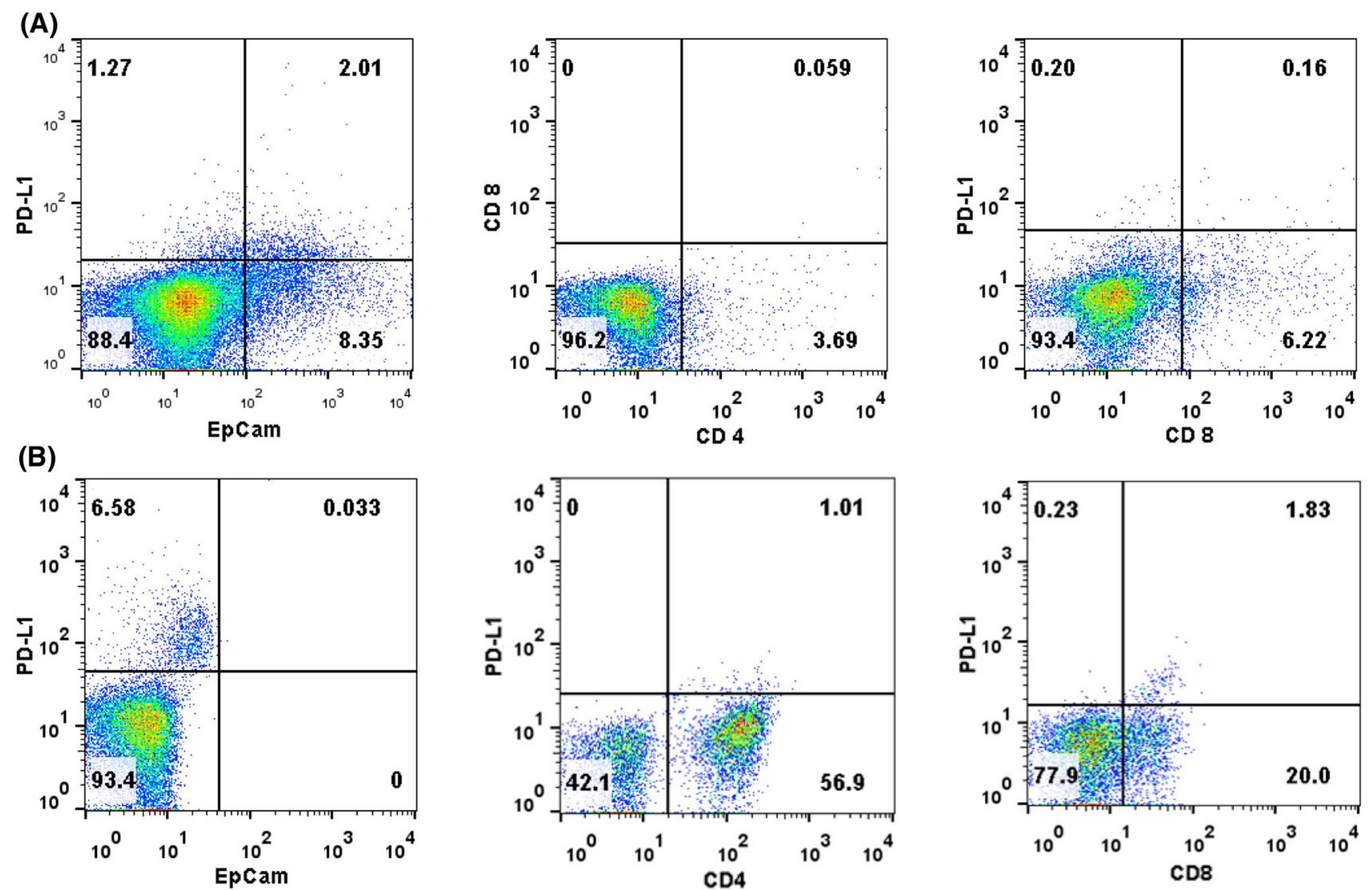

Fig. 3 Representative flow cytometry data for PD-L1 expression in human breast cancer a stage IV breast cancer b stage I breast cancer

modalities of assessment of PD-L1 expression or on the differential impact of PD-L1 expression on different subpopulations of TIL. In their study of 1,058 specimens, Droeser et al. [31] showed that there are different subpopulations of TIL depending on breast cancer subtype, with variable impact on the prognosis. It could thus be hypothesized that depending on the TIL subpopulations, expression of PD-L1 by tumor cells might have a differential impact on antitumor immunity.

In our analysis, the strongest association between PD-L1 and decreased OS was found in the basal-like intrinsic subtype. Since treatment options are limited for this subtype, our finding is of particular importance, and PD-1/PD-L1 blockade might prove to be a valid treatment option in these patients.

We recently reported the expression of PD-1 on TIL to be an independent negative prognostic factor in breast cancer [35]. Interestingly, we found a strong correlation between PD-L1 expression by tumor cells and the presence of PD- $1^{+}$TIL in our collective. This further suggests PDL1 expressed by tumor cells directly interacts with PD$1^{+}$TIL and in turn leads to attenuation of their antitumor activity. Our finding is also consistent with the hypothesis that therapeutic blockade of the PD-1/PD-L1 pathway might be a valid treatment approach in breast cancer.
Topalian et al. [25] analyzed PD-L1 protein expression in 42 tumor specimens of patients with various malignancies before treatment with anti-PD-1 antibody. They showed that only patients whose tumors expressed PD-L1 had an objective response to the anti-PD-1 therapy. Although these results were documented as preliminary and without statistical significance, they support the hypothesis that PD-L1 protein expression might constitute a predictive biomarker and help select patients for anti-PD-1/PD-L1 therapy. While there are ongoing clinical trials targeting the PD-1/PD-L1 signaling pathway [24-28], all of these studies include only a limited number of breast cancer patients.

As an interesting finding using flow cytometry, we found expression of PD-L1 on TIL in a small tumor and predominantly on cancer cells in a larger tumor. This might be an indication that as the tumor progresses PD-L1 is produced in an increasing fashion by tumor cells in order to evade the mounting antitumor immune response. Ghebeh et al. also found PD-L1 expression by TIL in $41 \%$ of their specimens, predominantly on CD4 + - T cells. Expression of PD-L1 on TIL might inhibit T-cell function by binding to PD-1 expressed on other TIL, as demonstrated by Seo et al. [56]. We could also detect immunohistochemical expression of PD-L1 on TIL in a small subset of cases 
(9.2\%), but in light of the low case number, the impact on prognosis was not analyzed.

We acknowledge that our study has some limitations such as the fact that the use of TMAs may not accurately represent PD-L1 protein expression due to intra-tumoral heterogeneity of expression. We therefore used whole tissue sections and flow cytometry to assess PD-L1 expression by tumor cells from the 4 breast cancer patients. In the two positive sections, there was no discernible intratumoral heterogeneity of PD-L1 expression, although this was a small collective (not encompassing patients whose tumors were included in the TMAs).

In addition, there are concerns regarding the reliability of immunohistochemical staining for PD-L1, due to the lack of a standardized staining and analysis protocol as well as the variety of antibodies. The commercially available rabbit-anti-human PD-L1 polyclonal antibody used here was validated using placental and lymph node tissue as positive controls. Moreover, using the same antibody, in 42 patients with HCC, Cariani et al. [57] not only similarly linked PD-L1 to a shorter OS, time to recurrence, but also found a positive association with TIL.

\section{Conclusion}

We are the first to show that the expression of PD-L1 in breast cancer is an independent negative prognostic factor. Our results suggest that it could serve as an important target for antibody-based immune therapies, especially in the basal-like intrinsic subtype where treatment options are limited. Further research investigating the effect of PD-L1/ PD-1 blockade in breast cancer is thus strongly needed.

Acknowledgments The authors wish to acknowledge the support of the Biostatistics Core, Siteman Comprehensive Cancer Center WUSM, and NCI Cancer Center Support Grant P30 CA091842.

We thank Professor Alexandar Tzankov for his contribution to the development of the PD-L1 immunohistochemical assay as well as his valuable insights and critical review of the manuscript. We also thank Professor Giulio Spagnoli for his support and critical evaluation of the manuscript.

Conflict of interest The authors declare that they have no conflict of interest.

\section{References}

1. Dermime $\mathrm{S}$ et al (2004) Vaccine and antibody-directed $\mathrm{T}$ cell tumour immunotherapy. Biochim Biophys Acta 1704(1):11-35

2. Bour-Jordan $\mathrm{H}$ et al (2011) Intrinsic and extrinsic control of peripheral T-cell tolerance by costimulatory molecules of the CD28/B7 family. Immunol Rev 241(1):180-205

3. Mueller DL, Jenkins MK, Schwartz RH (1989) Clonal expansion versus functional clonal inactivation: a costimulatory signalling pathway determines the outcome of $\mathrm{T}$ cell antigen receptor occupancy. Annu Rev Immunol 7:445-480

4. Liang SC et al (2003) Regulation of PD-1, PD-L1, and PD-L2 expression during normal and autoimmune responses. Eur $\mathrm{J}$ Immunol 33(10):2706-2716

5. Probst $\mathrm{HC}$ et al (2005) Resting dendritic cells induce peripheral CD8 + T cell tolerance through PD-1 and CTLA-4. Nat Immunol 6(3):280-286

6. Keir ME et al (2008) PD-1 and its ligands in tolerance and immunity. Annu Rev Immunol 26:677-704

7. Flies DB et al (2011) Blockade of the B7-H1/PD-1 pathway for cancer immunotherapy. Yale J Biol Med 84(4):409-421

8. Latchman $\mathrm{Y}$ et al (2001) PD-L2 is a second ligand for PD-1 and inhibits T cell activation. Nat Immunol 2(3):261-268

9. Freeman GJ et al (2000) Engagement of the PD-1 immunoinhibitory receptor by a novel B7 family member leads to negative regulation of lymphocyte activation. J Exp Med 192(7): $1027-1034$

10. Konishi J et al (2004) B7-H1 expression on non-small cell lung cancer cells and its relationship with tumor-infiltrating lymphocytes and their PD-1 expression. Clin Cancer Res 10(15): 5094-5100

11. Nomi $T$ et al (2007) Clinical significance and therapeutic potential of the programmed death-1 ligand/programmed death-1 pathway in human pancreatic cancer. Clin Cancer Res 13(7):2151-2157

12. Ohigashi Y et al (2005) Clinical significance of programmed death-1 ligand-1 and programmed death-1 ligand-2 expression in human esophageal cancer. Clin Cancer Res 11(8):2947-2953

13. Strome SE et al (2003) B7-H1 blockade augments adoptive T-cell immunotherapy for squamous cell carcinoma. Cancer Res 63(19):6501-6505

14. Iwai Y et al (2002) Involvement of PD-L1 on tumor cells in the escape from host immune system and tumor immunotherapy by PD-L1 blockade. Proc Natl Acad Sci USA 99(19):12293-12297

15. Dong $\mathrm{H}$ et al (2002) Tumor-associated B7-H1 promotes T-cell apoptosis: a potential mechanism of immune evasion. Nat Med 8(8):793-800

16. Thompson RH et al (2007) PD-1 is expressed by tumor-infiltrating immune cells and is associated with poor outcome for patients with renal cell carcinoma. Clin Cancer Res 13(6): 1757-1761

17. Thompson RH et al (2004) Costimulatory B7-H1 in renal cell carcinoma patients: indicator of tumor aggressiveness and potential therapeutic target. Proc Natl Acad Sci USA 101(49): 17174-17179

18. Blank C, Gajewski TF, Mackensen A (2005) Interaction of PD$\mathrm{L} 1$ on tumor cells with PD-1 on tumor-specific $\mathrm{T}$ cells as a mechanism of immune evasion: implications for tumor immunotherapy. Cancer Immunol Immunother 54(4):307-314

19. Morse MA et al (2005) Recent developments in therapeutic cancer vaccines. Nat Clin Pract Oncol 2(2):108-113

20. Rosenberg SA, Yang JC, Restifo NP (2004) Cancer immunotherapy: moving beyond current vaccines. Nat Med 10(9): 909-915

21. Li B et al (2009) Anti-programmed death-1 synergizes with granulocyte macrophage colony-stimulating factor-secreting tumor cell immunotherapy providing therapeutic benefit to mice with established tumors. Clin Cancer Res 15(5):1623-1634

22. Wang W et al (2009) PD1 blockade reverses the suppression of melanoma antigen-specific CTL by CD $4+\mathrm{CD} 25(\mathrm{Hi})$ regulatory T cells. Int Immunol 21(9):1065-1077

23. Mangsbo SM et al (2010) Enhanced tumor eradication by combining CTLA-4 or PD-1 blockade with CpG therapy. J Immunother 33(3):225-235 
24. Brahmer JR et al (2010) Phase I study of single-agent anti-programmed death-1 (MDX-1106) in refractory solid tumors: safety, clinical activity, pharmacodynamics, and immunologic correlates. J Clin Oncol 28(19):3167-3175

25. Topalian SL et al (2012) Safety, activity, and immune correlates of anti-PD-1 antibody in cancer. N Engl J Med 366(26): 2443-2454

26. Berger R et al (2008) Phase I safety and pharmacokinetic study of CT-011, a humanized antibody interacting with PD-1, in patients with advanced hematologic malignancies. Clin Cancer Res 14(10):3044-3051

27. Hamid $O$ et al (2013) Safety and tumor responses with lambrolizumab (anti-PD-1) in melanoma. N Engl J Med 369(2): 134-144

28. Brahmer JR et al (2012) Safety and activity of anti-PD-L1 antibody in patients with advanced cancer. $\mathrm{N}$ Engl $\mathrm{J}$ Med 366(26):2455-2465

29. Jemal A et al (2011) Global cancer statistics. CA Cancer J Clin 61(2):69-90

30. Liyanage UK et al (2002) Prevalence of regulatory T cells is increased in peripheral blood and tumor microenvironment of patients with pancreas or breast adenocarcinoma. J Immunol 169(5):2756-2761

31. Droeser R et al (2012) Differential pattern and prognostic significance of CD4+, FOXP3+ and IL-17+ tumor infiltrating lymphocytes in ductal and lobular breast cancers. BMC Cancer $12: 134$

32. Czerniecki BJ et al (2007) Targeting HER-2/neu in early breast cancer development using dendritic cells with staged interleukin12 burst secretion. Cancer Res 67(4):1842-1852

33. Ghebeh $\mathrm{H}$ et al (2008) FOXP3+ Tregs and B7-H1+/PD-1+ T lymphocytes co-infiltrate the tumor tissues of high-risk breast cancer patients: implication for immunotherapy. BMC Cancer 8:57

34. Ghebeh H et al (2006) The B7-H1 (PD-L1) T lymphocyteinhibitory molecule is expressed in breast cancer patients with infiltrating ductal carcinoma: correlation with important high-risk prognostic factors. Neoplasia 8(3):190-198

35. Muenst S et al (2013) The presence of programmed death 1 (PD1)-positive tumor-infiltrating lymphocytes is associated with poor prognosis in human breast cancer. Breast Cancer Res Treat 139(3):667-676

36. Goldhirsch A et al (2011) Strategies for subtypes-dealing with the diversity of breast cancer: highlights of the St. Gallen International Expert Consensus on the Primary Therapy of Early Breast Cancer 2011. Ann Oncol 22(8):1736-1747

37. Ghebeh H et al (2007) Expression of B7-H1 in breast cancer patients is strongly associated with high proliferative Ki-67expressing tumor cells. Int J Cancer 121(4):751-758

38. Hasan A et al (2011) Therapeutic targeting of B7-H1 in breast cancer. Expert Opin Ther Targets 15(10):1211-1225

39. Schalper K.A et al (2014) In situ tumor PD-L1 mRNA expression is associated with increased TILs and better outcome in breast carcinomas. Clin Cancer Res

40. McShane LM et al (2006) Reporting recommendations for tumor marker prognostic studies (remark). Exp Oncol 28(2):99-105

41. Bubendorf L et al (2001) Tissue microarray (TMA) technology: miniaturized pathology archives for high-throughput in situ studies. J Pathol 195(1):72-79
42. McCarty KS Jr et al (1985) Estrogen receptor analyses. Correlation of biochemical and immunohistochemical methods using monoclonal antireceptor antibodies. Arch Pathol Lab Med 109(8):716-721

43. Tapia $\mathrm{C}$ et al (2004) HER2 analysis in breast cancer: reduced immunoreactivity in FISH non-informative cancer biopsies. Int $\mathbf{J}$ Oncol 25(6):1551-1557

44. Perou CM et al (2000) Molecular portraits of human breast tumours. Nature 406(6797):747-752

45. Prat A, Perou CM (2011) Deconstructing the molecular portraits of breast cancer. Mol Oncol 5(1):5-23

46. Blows FM et al (2010) Subtyping of breast cancer by immunohistochemistry to investigate a relationship between subtype and short and long term survival: a collaborative analysis of data for 10,159 cases from 12 studies. PLoS Med 7(5):e1000279

47. Soliman H, Khalil F, Antonia S (2014) PD-L1 Expression Is Increased in a Subset of Basal Type Breast Cancer Cells. PLoS ONE 9(2):e88557

48. Velcheti V et al (2014) Programmed death ligand-1 expression in non-small cell lung cancer. Lab Invest 94(1):107-116

49. Taube JM et al (2012) Colocalization of inflammatory response with B7-h1 expression in human melanocytic lesions supports an adaptive resistance mechanism of immune escape. Sci Transl Med 4(127):127-137

50. Lipson EJ et al (2013) PD-L1 expression in the Merkel cell carcinoma microenvironment: Association with inflammation, Merkel cell polyomavirus and overall survival. Cancer Immunol Res 1

51. Droeser RA et al (2013) Clinical impact of programmed cell death ligand 1 expression in colorectal cancer. Eur J Cancer 49(9):2233-2242

52. Zang X, Allison JP (2007) The B7 family and cancer therapy: costimulation and coinhibition. Clin Cancer Res 13(18 Pt 1):5271-5279

53. Hino R et al (2010) Tumor cell expression of programmed cell death-1 ligand 1 is a prognostic factor for malignant melanoma. Cancer 116(7):1757-1766

54. Mu CY et al (2011) High expression of PD-L1 in lung cancer may contribute to poor prognosis and tumor cells immune escape through suppressing tumor infiltrating dendritic cells maturation. Med Oncol 28(3):682-688

55. Song $M$ et al (2013) PTEN loss increases PD-L1 protein expression and affects the correlation between PD-L1 expression and clinical parameters in colorectal cancer. PLoS ONE 8(6):e65821

56. Seo SK et al (2006) Co-inhibitory role of T-cell-associated B7$\mathrm{H} 1$ and $\mathrm{B} 7-\mathrm{DC}$ in the T-cell immune response. Immunol Lett 102(2):222-228

57. Cariani E et al (2012) Immunological and molecular correlates of disease recurrence after liver resection for hepatocellular carcinoma. PLoS ONE 7(3):e32493 